with the milk of a thyroidless goat and in three weeks' time was better.

Some of the milk was sent to St. Thomas's Hospital, where Dr. Hector W. G. Mackenzie kindly tried it on the following four cases.

CASE 12.-The patient was an unmarried woman, aged 21 years. The illness had commenced 17 months ago with swollen neck, tremors, and prominence of the eyes. On admission to hospital the patient was very thin and considerable exophthalmos was present. The thyroid gland was slightly enlarged ; there were tremors in the hands, the pulserate was 160 , and the patient's weight was 5 stones 8 pounds. She was ordered 35 ounces of the milk a day and she gradually gained in strength and in weight, the gain in the latter respect being 1 stone 2 pounds.

CASE 13. - The patient, aged 20 years, was unmarried. Two years previously to coming for treatment she had been an in-patient for eight months with marked exophthalmos, tremors of the hands, and a systolic murmur. She was treated with rodagen and $x$ rays to the thyroid and she got much better and gained 2 stones in weight. She was kept under observation and was readmitted a year later. All the symptoms of Graves's disease were well marked. She was treated with the milk from thyroidless goats and improved, gaining several pounds in weight and the pulse-rate falling.

CASE 14.-The patient was 20 years of age and unmarried. On admission there was enlargement of the thyroid gland with a thrill and the pulse-rate was 120 . There were tremors in the hands but no exophthalmos was present. She was treated with milk from a thyroidless goat, about a pint a day. She steadily improved, leaving in 34 days, and having gained 1 stone 3 pounds in weight, the hands being much less tremulous and the pulse-rate remaining about the same.

CASE 15.-The patient, an unmarried woman, aged 42 years, had suffered from Graves's disease for 14 years. Two years ago she had been an in-patient for ten months and had improved somewhat, but became worse again after returning home. She was readmitted and was treated with milk from thyroidless goats, but did not improve under the treatment.

It is satisfactory that none of the patients treated have so far died. It is not always so. On looking through the reports of one large hospital it is seen that there were in ten years five deaths, and at another hospital in the same time 11 deaths. Only two of the cases did not improve under the treatment (Cases 3 and 15), but the patient in Case 3 did improve while the milk could be given, and the condition in Case 15 was of long standing. It is, of course, possible that the improvement may have been due wholly, as no doubt it was in part, to the rest and good diet obtained in hospital, but the patient in Case 1 continued to lose ground under these conditions till the milk was given. Cases 2 and 11 were treated wholly at home, and in Cases 3 and 5 the patients became worse when the milk had to be stopped. Further, Dr. A. Gordon Gullan has reported good results from rodagen and thyroidectin. ${ }^{2}$

As the anti-body in the milk must be derived from the blood serum it would seem better to administer the dried serum and corpuscles from a thyroidless animal. But there is one point in favour of the milk: ordinary cow's milk must contain a certain amount of thyroid secretion, therefore we have either to give this or to cut ourselves off from an article of diet valuable for invalids : the use of milk from thyroidless animals relieves us from this dilemma. The reduction in the amount of thyroid secretion in the patient can also be brought about by the excision of part of the enlarged thyroid, and several surgeons have recently reported successful cases of this treatment. The operation has, however, been attended with a heavy mortality in the past; but in one case in which the patient was seriously ill with the usual symptoms, including paroxysmal attacks of palpitation and dyspncea, I removed the major part of the goitre and the patient was speedily and greatly benefited. ${ }^{3}$ If an operation is to be undertaken it should not be delayed until the patient is materially weakened by the disease. It is true that a certain number of cases recover under medical treatment, but what we have to avoid is not so much unnecessary operations as unnecessary deaths. Even if opera. tion is the best treatment for the majority of cases we still

2 The LanceT, Sept. 5th, 1908, p. 708.

3 Journal of Pathology and Bacteriology, vol. iii., 1896. require a medical treatment for the cases which are too mild for operation, for those patients who are too ill for operation, for those who decline operation, and for those cases which have been operated upon but which have not been completely cured.

Devonshire-place, $\mathrm{W}$.

\section{RADIOGRAPHY AS AN AID TO THE DIAGNOSIS OF DISEASES AND INJURIES OF THE SKULL AND BRAIN.}

BY ROBFRT KNOX, M.D. EDIN., M.R.C.S. ENG., L.R.C.P. LOND.,

MEDICAI OFFICER IN CHARGE OF ELECTRICAI DEPARTMENT, GREAT NOR'THERN CEN'TRAL HOSPITAL.

WIDE as are the applications of the Roentgen rays in the diagnosis of disease no more interesting field of investigation exists than that which comprises the subject stated above. This method of demonstration in relation to these diseases and injuries is of great importance. A radiographer should be a trained clinical observer and not merely a taker of radiographs, for on the correct interpretation of what one actually sees depends very largely the value of diagnosis by radiography. When Professor Roentgen first announced his great discovery medical men were eager to avail themselves of the advantages of this new aid to diagnosis. It was soon found that the Roentgen rays could be applied to all parts of the body and early efforts in the direction of cranial radiography showed that in the case of foreign bodies in the walls of the skull and cranial cavity the value of the new aid to diagnosis was great. Improved methods of application and instrumentation led to more accurate results until at the present time it is possible to show many lesions and injuries in the skull and its adjacent parts and in the brain itself. The position of foreign bodies can be clearly shown, fractures of the skull can be located, abscess of the brain should give a definite shadow, and the correct location and size may be shown in the skiagram. Tumours of the brain are also capable of being demonstrated; those which are attached to or have their origin in the bony walls or periosteum of the skull are readily recognised. The nature and consistence of cranial tumours determine the degree of the shadow which they are likely to give. A tumour having the same consistence as the brain tissue will not give a definite shadow, but a tumour of greater density than brain tissue should be capable of demonstration. In radiography of other parts of the body one has to show bones in relation to soft parts and, as a rule, this is comparatively easy, but in cranial radiography there has to be overcome the difficulty of dealing with a bony case consisting of two more or less thick walls with a space of several inches between. The early radiographs show merely an outline of the skull as a whole, with shadows of varying density which might or might not be capable of interpretation. Tater efforts have been more successful in showing the various component parts of the bony skull and the contents of the cranial cavity.

In order to get a good radiograph of the skull and its contents it is necessary to use a fairly powerful coil and to vary the exposure according to the amount of current used. Most of the coils in use at the present time give a large output of energy and if one can get a tube with a sufficient degree of penetration the demonstration of the skull and its contents is made comparatively easy. An $x$ ray tube with a spark gap of five or six inches is capable of penetrating the average skull. The time exposure will vary with the thickness of the subject and the rapidity of the photographic plate in use. Several of the skiagrams taken have been done with a portable apparatus and others with powerful stationary installations. The exposure varies from five seconds to five minutes and good results have been obtained with various exposures between those limits. Recently it has been possible to get good detail with even shorter exposures than five seconds, and no doubt in time an exposure of a fraction of a second mav be sufficient to overcome the resistance of the parts and yield good results.

The chief advantage of a short exposure lies in the fact that movements are reduced to a minimum and the resulting 
shadow is more clearly defined in consequence. While this which accurately represents the size of the organ radio. is of great value in the radiography of regions of the body where movement is constantly going on, such as in the lungs and heart, it naturally has not the same importance in the case of the skull, where we have a rigid bony case to deal with. Yet no doubt even here short exposures will yield better results.

Quite recently the exposure time has been cut down to fractions of a second. This has been achieved by means of Wehnalt breaks and a special time switch which automatically cuts off the current at the proper moment. The radiographs:so produced are remarkable for the clear definition of the parts taken, and one can readily understand why this graphed. In heart work this promises to be extremely useful and the result will probably be that orthodiagraphy will be largely superseded by the new method.

In order to appreciate and demonstrate the existence of abnormalities it is necessary to be familiar with the normal appearances of the parts under consideration. The skiagram shown below, represented in Fig. 1 (a reproduction of a radiograph of a normal skull showing the principal bony points), was taken from a normal subject and an examination of its details will show the chief bony points which it is necessary to note for comparison when considering possible abnormalities.

FrG. 1



Skiagram of a normal head, showing appearances at the base of the brain. Note especially the two portions of the pituitary body, the anterior portion being situated in the sella turcica (APE) and the posterior portion behind it (PPB). A, Condyle of lower jaw, bone. J, Groove for blood-vessels. I, Anterior clinoid process.

should be so. The exposure is shortened by increasing to an enormous extent the amount of current passed through a tube. The tabe will stand the strong current only for a short time, but sufficiently long to enable the rays to penetrate the thickest portions of the body and give good results. The important point about the new development lies in the very short duration of the exposure and the consequent elimination of the effects of voluntary and involuntary movements on the part of the subject. In the case of children this is a very important factor in the obtaining of good radiographs. Another advantage is that the patient can be placed at a greater distance than at present. At about six feet from the anode of the tube a shadow can be obtained
The points to observe are the levels of the base of the skull in relation to the exterior, the clinoid processes with the sella turcica between, the relations of the chief sutures to the various levels, and the well-marked grooves in the inner table of the skull for blood vessels. A clearly-defined shadow is thrown by the pituitary body situated in the sella turcica. The three levels of the base of the skull are well shown. This radiograph was taken with a 12-inch intensified induction coil by Watsons and a Mote magnetic break on a voltage of 240. The tube used was a heary anode by Muller and the equivalent spark gap was about five inches. The exposure was a long one of about three minutes. A simple diaphragm compressor was used to cut 
off secondary radiations. An Ilford special $x$ ray plate was the whole skull and showed an obscuration of detail in the used. The developer was pyro soda. The development of region of the sella turcica. It was noticed that the anterior the plate took half an hour.

clinoid processes were barely perceptible while the posterior

Fig. 2 shows the appearance of the skull in a case of were obliterated.

cerebral tumour: a chiasma lesion of fairly large size. The radiogram which it represents was taken with a diaphragm between the tube and the subject to cut off secondary a radiations. The case was sent to me by Dr. W. Mailer of Muswell-hill, and Dr. Purves Stewart has kindly supplied me with the following particulars :-

The patient was a man, aged 35 years, who complained of persistent The patient was a man, aged 35 years, who complained of persistent
drowsiness of several months' duration. A month before coming under

The appearances seen in the skiagram can be interpreted as follows: A well-defined shadow occupies the sella turcica and extends backwards to the posterior limit of the petrous temporal bone; it is of varying density, having light and dark areas in its midst. The anterior clinoid processes are ill defined and appear to be spread out laterally. The posterior processes are not to be seen. The shadow of the tumour fades away into the brain substance.

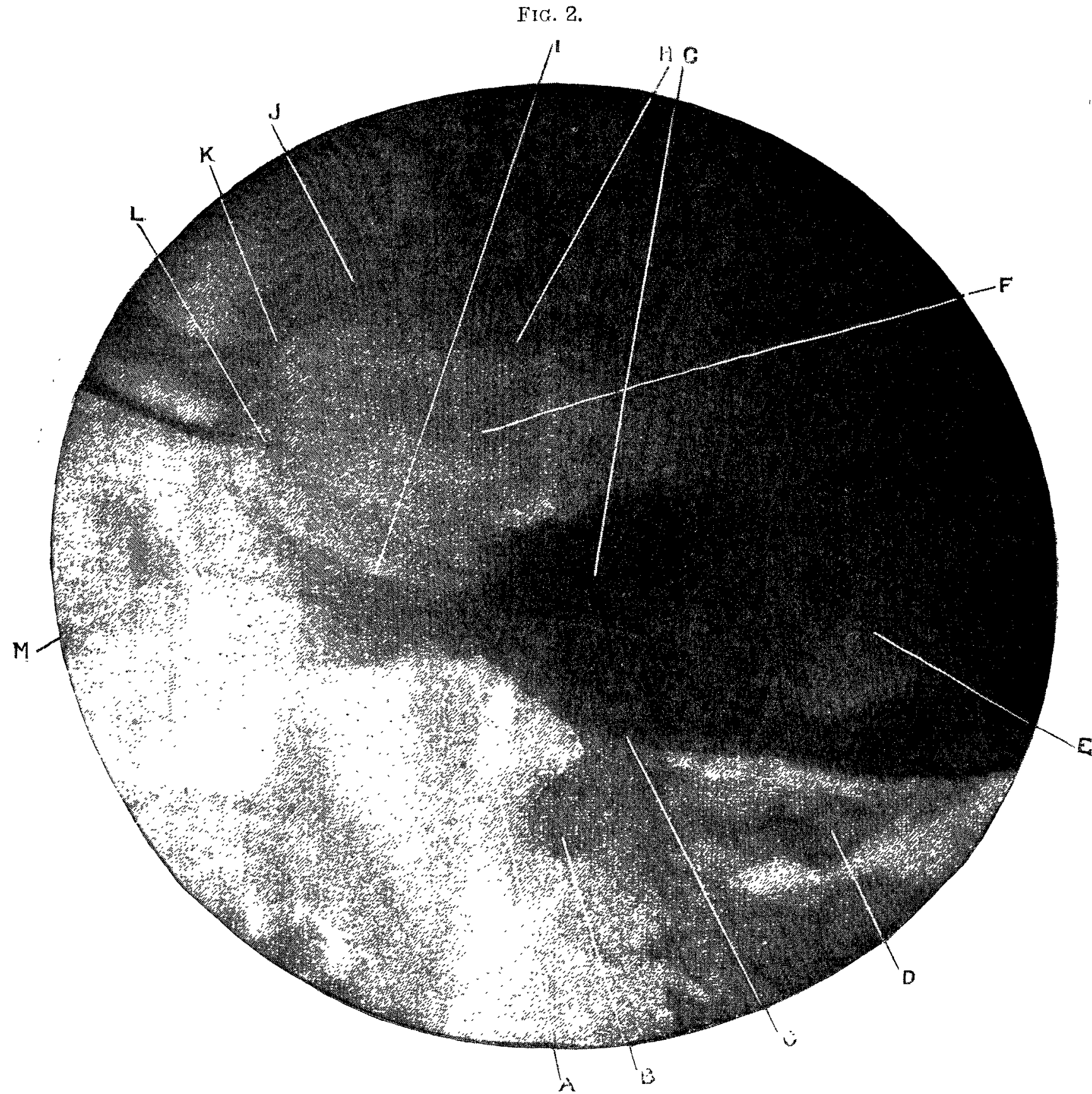

Skiagram from a case of tumour at the base of the brain. Note obliteration of definition of sella turcica. A, Condyle of lower jaw. B, Body of vertebra. C, Mastoid process. $\mathrm{n}$, Spinous process of vertebra. $\mathrm{F}$, Cerebellum. F, Shadow of tumour. G, Petrous portion of temporal bone. H, Upper limit of shadow of tumour. I, Lower limit of shadow of tumour. J, Groove for blood-vessel. $\mathbf{K}$ and $\mathbf{L}$, Anterior clinoid process, ir, Sphenoidal fissure.

observation he had had sudden unconsciousness with convulsions. Next day he noticed the right eye blind. On examination he was found to have total blindness of the right eye with temporal hemianopia of the left. The condition was characteristic of chiasma lesion. The dises were pale. The knee- and ankle-jerks were normal and the plantar reflexes plantar. $X$ rays showed enlargement of the pituitary fossa. A deep-seated fronto-occipital headache supervened and an operation was performed by Mr. C.A. Ballance to try to remove the growth. It was found inoperable. The patient died and at the postmortem examination a large growth was found at the base extending from the chiasma backwards to the pons and indenting the base of the brain.

The $x$ ray examination of this case was particularly in structive. The patient had an average sized head which was rounded and wide. He was strongly built and had well developed cranial bones. The early radiographs included
The examination of the brain offers the greatest difficulties in the Roentgen diagnosis of diseases. Dr. Paul Regnier and Jules Gloner have published a paper on this subject which is full of useful information. Dr. D. Walsh summarises their results as follows :-

Their main conclusions are that it is possible to superimpose upon the photographic plate the outline of the cranial sutures and of the cerebral traced, the relations of the venous sinuses, especially of the lateral sinus, traced, the relations of the venous sinuses, especially of the lateral sinus, to the skull can be defined both in the child and the adult. The extremely variable form of the cavities and bony cells of the bony
sinuses can be studied. The exact position of the three levels of the base of the skull can be ascertained with regard to the outer skull.

The exposures they gave were long ones, from 10 to 40 
minutes. Such exposures are not safe in the living subject. None of the exposures in the cases quoted in this paper exceeded four minutes. Good results can be obtained with an exposure of five seconds and in view of the recent developments one is safe to predict that in the future it will be possible to get a good radiograph from the living subject with a second's exposure and possibly with one of a tenth of a second. The advantages of such a short exposure cannot be over-estimated. With such short exposures it will be possible to clearly outline the bony points of the skull, the grooves for the blood-vessels in the walls of the skull, and possibly the outlines of the chief parts of the brain. Abscesses in the brain might be shown, and it might even be possible to locate hæmorrhage of any considerable size. Tumours are capable of being demonstrated and located. The aid to diagnosis and localisation of such tumours will be great.

Dr. Walsh, in his book "The Röntgen Rays in Medical Work," quotes several interesting cases by various workers and describes methods for the localisation of foreign bodies in the brain. By a simple method which he describes it is, as he says, possible to locate with the tube in two positions by bringing an opaque object into line with the foreign body and then marking the entry and exit of that line on both sides of the skull, the point of intersection being the site of the foreign body. In a case of bullet in the brain a rough method of localisation was adopted by me to which I will refer. A thin piece of wire was placed around the head as nearly as possible in line with the lower and outer angles of the orbits and the mastoid processes. Another piece was carried over the vertex of the skull in line with the anterior edges of the auricles. The patient was then placed on his side with the plate underneath his head, and a radiograph was obtained in this position. The position was then reversed and one taken from the opposite side. A third negative was obtained in the antero-posterior position. On comparing the three plates it was seen that a large fragment of the bullet was lodged in the occipital region immediately underneath the bone and on the right side of the brain. The surgeon who operated on this case was able to locate the bullet accurately and made his trephine wounds in the skull in such a position that when the bone was removed the portion of bullet could be felt with a probe in the position indicated by the radiographs. An instrument capable of being adjusted to fit the skull of any individual could readily be devised of such a nature that the various portions of the skull and brain could be divided into sections and when used in radiography would show where a foreign body was situated.

In a case of a foreign body in the right eyeball we were able to see from the radiograph that the fragment of steel was clearly in the eyeball and from its size it was evident that the function of the organ must necessarily have been destroyed. The eyeball and foreign body were removed by operation. For purposes of localising foreign bodies in the eyeball Dr. J. Mackenzie Davidson has devised a useful apparatus which should be applied in all cases where doubt exists as to the exact position of a foreign body in the orbit. The importance of determining whether a foreign bodv is in the eyeball or in the orbit is, of course, obvious, and careful radiographic examination should be made in all such cases before an operation is decided upon.

The examination of the ear by radiography may be helpful in cases of disease. Abscesses in the mastoid region should be capable of demonstration. The frontal sinuses and antrum can be examined by taking negatives in various positions, and, where possible, a probe passed into these cavities before the radiograph is taken will help to clear up the diagnosis in diseases of those parts.

Shepherd's-hill, N.

Shrewsbery Eye Hospital - At the annual meeting of the Shrewsbury Eye, Ear, and Throat Hospital, an institution which was established as long ago as 1818, the honorary ophthalmic surgeon, Mr. C. G. Russ Wood, commented on the large increase in the number of children who required spectacles. The increase is, no doubt, more apparent than real and is due to the inspection of school children which is being carried out in Shropshire and the adjoining Welsh counties from which the patients of the hospital are drawn. During the past year an entirely new system of drainage has been carried out at the hospital and a new out-patient operating theatre has been erected. In spite of the unusual expenditure thus entailed the balance-sheet was a satisfactory one.

\section{SOME REMARKS ON TUBERCULIN THERAPY.}

By A. J. LANDMAN, M.D., Ch.B. VICT.,

LATE HOUSE PHYSICIAN, GENERAL INFTRMARY, LEEDS ; AND RESTDEN MEDICAL OFFICER, IDA AND ROBER'T ARTHINGTON HOSPITAIS, COOKRIDGE.

THE growth and increasing importance of vaccine therapy at the present time is generally recognised. The opsonic method of Wright has established this mode of treatment on a scientific basis. The brilliant results obtained by this treatment in staphylococcic infections have led to the hope that equally good results might be obtained in other infections. Thus the vaccine treatment of tuberculosis originally introduced by Koch has again been brought into prominence, and tuberculin, which had fallen into disrepute, was again taken up as a curative agent. Administered according to the principles laid down by Wright, tuberculin has proved free from the dangers formerly associated with it, and in many cases good results have been obtained. While the final verdict cannot yet be given as to its efficacy it may be said that the results obtained have been encouraging.

Since the introduction of tuberculin by Koch ${ }^{1}$ about 17 years ago for the curative treatment of tuberculous disease there have been marked fluctuations in its popularity in the medical world. It was received with enthusiasm and subjected to extensive clinical trials. But it was not destined to fulfil the hopes entertained of its utility. Many observers reported that it was a dangerous remedy and it soon fell into disrepute. The report of the Brompton Hospital for Consumption, 1892, stated that tuberculin did not favourably influence the course of the disease in the majority of cases. The reaction against it was probably excessive. It proved disappointing for several reasons. The doses recommended by Koch were too high, and it was used in unsuitable cases. But the fact remained that it was practically abandoned as an agent, if not positively harmful, at least incapable of effecting the good claimed for it. It was no longer looked upon as a curative agent, but the febrile reaction which it produced in a tuberculous individual was reganded as of value in diagnosis. In veterinary practice it has found a permanent value as a means of recognising concealed tuberculosis, and recently Calmette, Wolff-Fisner, and von Pirquet have utilised it successfully for human diagnosis.

The dangers associated with the administration of old tuberculin led Koch and his pupils to continue their researches in order to produce a safer tuberculin. In 1897 Koch ${ }^{2}$ announced that he had prepared a new tuberculin which retained the curative and immunising power of the old one, but did not produce any dangerous febrile reaction, and no ill-consequence resulted from its use in tuberculous cases. The dose recommended by Koch was 1-500th milligramme, gradually increased to 20 milligrammes. The introduction of the new tuberculin T.R. caused some renewal of interest in the subject for a time, but in England it did not seem to gain any general hold.

Recently Koch has introduced a new tuberculin B.F. (Bacillen-Emulsion). Assuming that tuberculin acts in the same way as the other vaccines, the preparation B.E. should prove the most satisfactory of all the tuberculins. During the last few years there has been a considerable revival of interest in the tuberculin treatment. This has been due in England chiefly to the researches of Wright on opsonins and the application of his opsonic index determinations to the treatment placing it in a line with the treatment by other vaccines. But it must not be forgotten that many physicians have continued to use tuberculin ever since its introduction quite independently of Wright's work on the subject. The detailed method associated with Wright's name for the determination of the opsonic index is not an essential part of the therapeutic application of tuberculin. Previously the tuberculin treatment has been regulated by other clinical signs, including pulse, temperature, patient's aspect, local symptoms and signs, and prolonged experience justifies the statement that these clinical signs will suffice, apart from opsonic index determinations. The complexity of the technique

1 R. Koch : Mittheilungen über ein Heilmittel gegen d. Tuberkulose, Deutsche Medicinische Wochensehrift, No. 14, 1890 ; Mittheilungen ueut das Tuberkulin, ibid., Jan. 15th, 1891, and Oct. 22nd, 1891. 